\title{
Publisher Correction: Coherent diffractive imaging of single helium nanodroplets with a high harmonic generation source
}

\section{Daniela Rupp et al.}

Correction to: Nature Communications https://doi.org/10.1038/s41467-017-00287-z, Article published online 08 September 2017

In the original version of this Article, the affiliation for Luca Poletto was incorrectly given as 'European XFEL GmbH, Holzkoppel 4, 22869 Schenefeld, Hamburg, Germany', instead of the correct 'CNR, Istituto di Fotonica e Nanotecnologie Padova, Via Trasea 7, 35131 Padova, Italy'. This has now been corrected in both the PDF and HTML versions of the Article.

Published online: 16 January 2018

\begin{abstract}
(c) (i) Open Access This article is licensed under a Creative Commons Attribution 4.0 International License, which permits use, sharing, adaptation, distribution and reproduction in any medium or format, as long as you give appropriate credit to the original author(s) and the source, provide a link to the Creative Commons license, and indicate if changes were made. The images or other third party material in this article are included in the article's Creative Commons license, unless indicated otherwise in a credit line to the material. If material is not included in the article's Creative Commons license and your intended use is not permitted by statutory regulation or exceeds the permitted use, you will need to obtain permission directly from the copyright holder. To view a copy of this license, visit http://creativecommons.org/licenses/by/4.0/.
\end{abstract}

(C) The Author(s) 2018

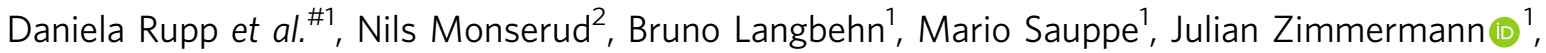
Yevheniy Ovcharenko ${ }^{1,3}$, Thomas Möller ${ }^{1}$, Fabio Frassetto ${ }^{4}$, Luca Poletto ${ }^{4}$, Andrea Trabattoni ${ }^{4,5}$, Francesca Calegari ${ }^{5,6}$, Mauro Nisoli ${ }^{6,7}$, Katharina Sander ${ }^{8}$, Christian Peltz $^{8}$, Marc J. Vrakking ${ }^{2}$, Thomas Fennel ${ }^{2,8} \&$ Arnaud Rouzée ${ }^{2}$

${ }^{1}$ Institut für Optik und Atomare Physik, Technische Universität Berlin, Hardenbergstraße 36, 10623 Berlin, Germany. ${ }^{2}$ Max-Born-Institut für Nichtlineare Optik und Kurzzeitspektroskopie, Max-Born-Straße 2A, 12489 Berlin, Germany. ${ }^{3}$ European XFEL GmbH, Holzkoppel 4, 22869 Schenefeld, Hamburg, Germany. ${ }^{4}$ CNR, Istituto di Fotonica e Nanotecnologie Padova, Via Trasea 7, 35131 Padova, Italy. ${ }^{5}$ Center for Free-Electron Laser Science, DESY, Notkestr. 85, 22607 Hamburg, Germany. ${ }^{6}$ CNR, Istituto di Fotonica e Nanotecnologie Milano, Piazza L. da Vinci 32,20133 Milano, Italy. ${ }^{7}$ Department of Physics, Politecnico di Milano, Piazza L. da Vinci 32, 20133 Milano, Italy. ${ }^{8}$ Institut für Physik, Universität Rostock, Albert-Einstein-Straße 23, 18059 Rostock, Germany. The original article can be found online at https://doi.org/10.1038/s41467-017-00287-z. 